\title{
Validity of current force fields for simulations on boron nitride nanotubes
}

\author{
T.A. Hilder ${ }^{1}$ R. Yang ${ }^{2}$ V. Ganesh ${ }^{2}$ \\ A.P. Rendell ${ }^{2}$ S.-H. Chung ${ }^{1}$ \\ ${ }^{1}$ Computational Biophysics Group, Research School of Biology, Australian National University, ACT 0200, Australia \\ ${ }^{2}$ Computer Science, Faculty of Engineering and Information Technology, Australian National University, ACT 0200, Australia \\ ${ }^{3}$ Australian National University Supercomputer Facility, Australian National University, ACT 0200, Australia \\ E-mail: tamsyn.hilder@anu.edu.au
}

\begin{abstract}
Past molecular dynamics (MD) studies of boron nitride nanotubes (BNNTs) have used van der Waals parameters from generic force fields, combined with various values for the partial charges on the boron and nitrogen atoms. This study explores the validity of these parameters by first using quantum chemical packages Car-Parrinello molecular dynamics (CPMD) and Gaussian to compute partial charges for isolated and periodic BNNTs, both with and without water. Then in order to test the accuracy of the molecular mechanics force field using our computed charges, the authors calculate the interaction energy between each water molecule in a hydrated nanotube with the nanotube itself using two methods: first using a quantum chemical calculation, and secondly using the molecular mechanics force field. The authors show that in order to obtain satisfactory agreement in the interaction energies the boron and nitrogen Lennard-Jones parameters must be adjusted from their usual values. Modified Lennard-Jones parameters and partial charges, obtained by fitting, are presented as candidates for future MD simulations of hydrated BNNTs.
\end{abstract}

\section{Introduction}

Boron nitride nanotubes (BNNTs) are receiving increasing attention for applications such as filtration $[1,2]$, storage materials [3], electronics, biosensors and as molecular transporters to deliver biological molecules into living cells [4]. They share a similar structure to carbon nanotubes [5], but exhibit improved properties such as high resistance to oxidation. Recent molecular dynamics (MD) simulations indicate that BNNTs may have superior water permeation properties as compared to carbon nanotubes of similar diameter and length $[6,7]$. However, unlike the popular carbon nanotube, little effort has been devoted to examining the validity of their force fields.

Arguably, the most important terms in any force field that is used to simulate the behaviour of BNNT will be the partial charges and van der Waals parameters associated with the boron (B) and nitrogen $(\mathrm{N})$ atoms. These will determine how the nanotube interacts with other systems, and particularly with atoms or molecules seeking to pass through it. Furthermore, to capture the electronegativity difference between the boron and nitrogen atoms, the force field is likely to require that the charges on the boron and nitrogen atoms are significant. This is an important difference compared to carbon nanotubes, where charges on the carbon atoms arise predominantly from end defects, and are near zero elsewhere $[8,9]$.

Like carbon nanotubes [10,11] conjugated $\pi$ bonding would be expected to make BNNTs polarisable. In a recent study of carbon nanotubes, it was found that polarisation of the carbon nanotube by water can account for up to $8 \%$ of the total interaction energy [12]. For BNNT polarisation might be expected to make a larger contribution to the interaction energy, not least because of the larger charges on the boron and nitrogen atoms.

Most force fields do not, however, include explicit polarisation terms. Instead they assume that polarisation 
effects can be implicitly accounted for as part of the overall parameterisation of the force field. For BNNT it is by no means clear that this will work.

When developing a force field partial charges on the atoms are usually determined by performing a series of quantum chemical computations to evaluate the electrostatic potential at various points in the system. Charges are then assigned to atom centres in order to best fit the observed potential. Example methods include CHelpG [13] and Merz Kollman (MK) [14, 15]. Occasionally partial charges are obtained using a population analysis scheme, such as Mulliken [16], although this is considered to be less reliable because it generally leads to charges that do not adequately reproduce the electrostatic potential calculated from the quantum mechanical wavefunction. In contrast to the various methods used for determining partial charges, the van der Waals parameters are usually just taken from a generic force field with little or no adjustment.

In early molecular simulations on the permeation of water through BNNT [6] the boron and nitrogen atoms were given no partial charge, thus neglecting the effect of electrostatics completely. This is clearly unphysical, given the electronegativity difference mentioned above. This view is supported by the quantum chemical calculations of Nirmala and Kolandaivel [17] that report significant charges on both the boron and the nitrogen atoms. In their work Nirmala and Kolandiavel used a $6-31 G^{* *}$ basis set, B3LYP density functional theory (DFT) and a variety of population analysis-based schemes. Only isolated nanotubes without water were considered, with one end of the nanotube capped with hydrogen atoms in an attempt to simulate the environment of a bulk nanotube. Interestingly, they report large differences of $\sim 0.5 e$ between the charges computed using Mulliken and natural population analysis (NPA) [18] schemes at the open end of the tube, but much smaller difference of $\sim 0.2 e$ at the hydrogen capped end. From the results given it is not clear which, if any, of their various charge values should be used in a classical force field.

In more recent work Won and Aluru [7] have used the CHelpG scheme to derive partial charges on the boron and nitrogen atoms for use in force fields by performing B3LYP/6-31G** quantum chemical calculations on the $(5$, 5) BNNT. They consider systems both with and without water reporting values of $\pm 0.4 e$ and $\pm 1.05 e$, respectively. They found that the partial charges obtained with water improve the wetting behaviour, but that there was also a slight decrease in the diffusion coefficient because of the formation of hydrogen bonds between the water and nitrogen atoms. This observation has since been extended by Hilder et al. [1] who showed that different charges significantly affected the conduction of water molecules through a BNNT membrane.

From existing work it is apparent that the charge computed on the boron and nitrogen atoms is highly sensitive both to the means of calculation, that is population analysis or fitting, and the presence of water. It is argued that charges obtained via fitting are more accurate than those obtained using population analysis, but this may not be true if most of the grid points used to fit the potential are located outside the nanotube, yet it is the electrostatic potential inside the nanotube that is most important for permeation of water or charged particles through the nanotube. Also, in the work of Won and Aluru [7], it is not clear what charges, if any, were allowed on the water molecules as part of the fitting process. Noting that the quantum chemical and molecular mechanics charges for water (e.g. TIP3P) are usually different, this may give rise to another source of error.

To the authors' knowledge none of the previous studies on BNNT have considered the van der Waals parameters. Typically, Kang and Hwang [3] and Won and Aluru [6, 7] have used the van der Waals parameters for boron and nitrogen atoms given by either Mayo et al. [19] or Rappé et al. [20] that were taken from the DREIDING generic force field and Universal force field, respectively. However, it should be noted that BNNTs are different to most commonly found compounds containing boron and nitrogen. Therefore the validity of the van der Waals parameters used previously is highly questionable.

Here the authors attempt to validate the force field parameters used to represent BNNT. First the charges on the boron and nitrogen atoms in the BNNT are computed using a variety of different methods with and without the presence of water molecules. Using these charge values the interaction energy of water with BNNT obtained from a classical force field with values obtained using quantum methods are compared. Finally, an alternative approach is considered where both the charge and van der Waals parameters in the force field are fitted directly to the water/nanotube interaction energies. With the respect to the latter, it is important to note that the aim is to determine whether it is possible to determine an alternative set of force field parameters that can reasonably describe the specific case of water interacting with BNNTs, not to determine a new set of generally applicable parameters.

\section{Computational details}

The force field calculations reported here were performed using the NAMD [21] MD program. In this program the van der Waals $\left(E_{\mathrm{LJ}}\right)$ and Coulomb interaction energies $\left(E_{\text {elec}}\right)$ are given by

$$
\begin{gathered}
E_{\mathrm{LJ}}=\sqrt{\varepsilon_{i} \varepsilon_{j}}\left[\left(\frac{\left(\sigma_{i}+\sigma_{j}\right) / 2}{R_{i j}}\right)^{12}-2\left(\frac{\left(\sigma_{i}+\sigma_{j}\right) / 2}{R_{i j}}\right)^{6}\right], \\
E_{\text {elec }}=\frac{C q_{i} q_{j}}{\varepsilon_{0} R_{i j}}
\end{gathered}
$$


where $\varepsilon_{i}$ and $\sigma_{i}$ are the usual empirical Lennard-Jones parameters associated with atom $i, R_{i j}$ is the distance between the atoms $i$ and $j, q_{i}$ and $q_{j}$ are their charges, $C$ is Coulomb's constant and $\varepsilon_{0}$ is the dielectric constant. This Letter considers the values of $\varepsilon, \sigma$ and $q$ that should be used for boron and nitrogen in simulations of BNNT.

To perform the quantum computations two different approaches were used. The first was based on the CarParrinello molecular dynamics (CPMD) code [22]. This is a plane-wave pseudopotential implementation of DFT. In principle, CPMD can perform the same type of simulations as NAMD, but using a much more expensive DFT interaction potential that necessitates a much smaller timestep. The second approach used the Gaussian 03 code (G03) [23]. In contrast to CPMD, this program uses a localised basis set, supports a larger variety of quantum mechanical methods and does not require the system to be periodic or to use pseudopotentials.

For the CPMD computations, the BLYP generalised gradient approximation functional was used, with a fully nonlocal norm-conserving Troullier-Martins pseudopotentials in the Kleinman-Bylander separable form. The planewave basis set used a kinetic energy cutoff of $80 \mathrm{Ry}$. (A larger cutoff energy of 90 Ry changes the total energy by less than $0.02 \%$.) For the Gaussian computations both the BLYP and B3LYP DFT methods were used. All Gaussian computations used a $6-31 \mathrm{G}^{*}$ basis set, with interaction energies corrected to account for basis set superposition errors using the counter poise method [24].

It is widely recognised that the BLYP and B3LYP functionals poorly describe long-range dispersive-type interactions. Recently, various empirical corrections have been proposed to remedy this deficiency. In this Letter, the authors used the Grimme dispersion correction [25] that is available in CPMD. This is defined as

$$
E_{\mathrm{disp}}=-s_{6} \sum_{i=1}^{N_{\mathrm{at}}-1} \sum_{j=i+1}^{N_{\mathrm{at}}} \frac{C_{6}^{i j}}{R_{i j}^{6}} f_{\mathrm{dmp}}\left(R_{i j}\right)
$$

where $f_{\mathrm{dmp}}=1 /\left[1+\exp \left(-d\left(R_{i j} / R_{r}-1\right)\right)\right], R_{r}$ is the sum of van der Waals radii (where $R_{0}=1.485 \AA$ for boron and $R_{0}=1.397 \AA$ for nitrogen), $N_{\mathrm{at}}$ is the number of atoms in the system, $C_{6}^{i j}$ is the dispersion coefficient for atom pair $i j$ (3.13 $\mathrm{J} \mathrm{nm}^{6} / \mathrm{mol}$ for boron, $1.23 \mathrm{~J} \mathrm{~nm}^{6} / \mathrm{mol}$ for nitrogen), $s_{6}$ is a global scaling factor that depends on the density functional used (in this case 1.2), $d$ is equal to 20 and $R_{i j}$ is the interatomic distance. Partial charges on the boron and nitrogen atoms were derived using the MK [15] scheme as implemented in CPMD, and both the CHelpG [13] and MK schemes as implemented in G03.

Like carbon nanotubes, BNNT are defined by their chiral vector $\boldsymbol{C}=(n, m)$. In this Letter results for the $(5,5)$ armchair-type tube are presented. An initial geometry for this system was constructed from a hexagonal array of alternating boron and nitrogen atoms rolled up to form a tubular structure similar to a carbon nanotube $[5,26]$. This was done using visual molecular dynamics (VMD) [27] for a nanotube fragment that contained 120 atoms, with an experimental boron to nitrogen bond distance of $1.446 \AA$ [5]. The length of this fragment defined as the $z$ dimension of the unit cell used in the CPMD computations to be $14.76 \AA$, whereas $x$ and $y$ dimensions were set to be $15 \AA$ each. ( $15 \AA$ was found to be sufficiently large to avoid spurious image effects on the total energy.) This system was then relaxed using CPMD, but keeping the cell fixed. The resulting optimised structure forms the basis for the 'without-water' comparisons given later in this paper. Similar to previous work [28], the nitrogen atoms in this optimised structure shift outwards relative to the boron atoms by approximately $0.07 \AA$.

To generate nanotube structures containing water, one unit cell from the CPMD optimised structure was used in an MD simulation with water reservoirs at either end of the nanotube. After equilibration it was found that there were on average six water molecules contained within the nanotube, and that these formed a linear chain down the centre of the nanotube. One of these water configurations was selected at random and optimised using CPMD. This structure forms the basis for the 'with-water' comparisons given later in this Letter, and is illustrated in Fig. 1.

\section{Results and discussion}

The partial charges on the boron and nitrogen atoms computed using a variety of methods are given in Table 1. Both the BNNT structures with and without water are used. The Gaussian computations use a single cell with the same geometry and with no capping atoms. The charges reported for CPMD are an average over all atoms in a cell. In the case of Gaussian, however, an average of charges on atoms in the central four rings is used in order to avoid boundary effects. (Note that not accounting for all atoms when computing the average charges gives rise to a slight imbalance in partial charge between the boron and nitrogen atoms.) Also shown are values taken from previous studies of Won and Aluru [7] and Nirmala and Kolandaivel [17].

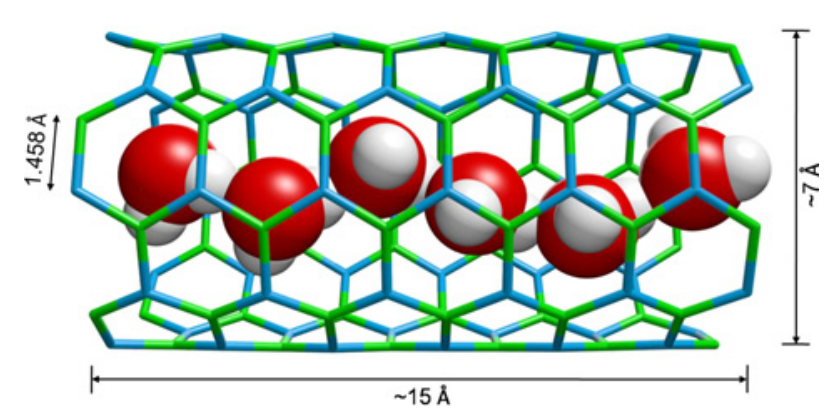

Figure 1 CPMD optimised geometry of a $(5,5) B N N T$ with a single-file chain of size water molecules using BLYP functional 
Table 1 Partial charge $(e)$ on B and N atoms of the $(5,5)$ BNNT, determined from CPMD and Gaussian computations, using different functionals and charge analysis schemes and compared to previous studies [7, 17]

\begin{tabular}{|l|c|c|c|c|c|}
\hline \multirow{2}{*}{ Method } & \multirow{2}{*}{ Charge scheme } & \multicolumn{2}{c|}{ Without water } & \multicolumn{2}{c|}{ With water } \\
\cline { 3 - 6 } & & $\mathrm{B}$ & $\mathrm{N}$ & $\mathrm{B}$ & $\mathrm{N}$ \\
\hline CPMD (BLYP) & MK & 0.350 & -0.350 & 1.296 & -1.297 \\
\hline \multirow{4}{*}{ G03 (BLYP) } & MK & 0.387 & -0.389 & 0.984 & -0.959 \\
& CHelpG & 0.382 & -0.405 & 1.075 & -1.051 \\
\cline { 2 - 6 } & CHelpG, OuterG & 1.037 & -1.028 & 0.893 & -1.372 \\
\cline { 2 - 6 } & ChelpG, InnerG & 0.145 & -0.139 & 0.972 & -0.973 \\
\cline { 2 - 6 } & CHelpG & 0.403 & -0.407 & 1.144 & -1.118 \\
\hline G03 (B3LYP) & CHelp G & 0.40 & -0.40 & 1.05 & -1.05 \\
\hline [7] & Mulliken, NPA & {$[0.57,1.17]$} & {$[-0.55,-1.17]$} & - & - \\
\hline [17] & a & &
\end{tabular}

Results for [17] represent a range of values. InnerG and OuterG represent grid points inside and outside the BNNT, respectively

Considering first the nanotube 'without-water'. The values obtained when using CPMD and the BLYP functional $( \pm 0.35 e)$ are slightly less than those obtained using G03 with the same functional and method $( \pm 0.39 e)$. The difference reflects the combined effect of moving from an infinite periodic system to an isolated fragment and from a plane-wave basis set to a localised basis set. Using CHelpG with G03 (the default grid-based scheme) gives only a slight difference in partial charges in comparison to MK.

To explore the effect of grid points, the CHelpG charges were recomputed with all points inside the tube removed; this attempts to model the grid that must be used when water molecules are present within the tube. A second computation was also performed with the grid points positioned exclusively inside the tube. Although the default grid consists of around 40000 points, there are about 34000 and 6000 points outside and inside the tube, respectively. The large difference in charges computed using the different grids clearly indicates the need to be careful when using grid-based methods for deriving partial charges for systems such as BNNT. Use of the hybrid B3LYP functional has only a small effect on the average charge values, suggesting that the requirement of the CPMD code to use a pure DFT functional does not lead to significantly different results.

The partial charges computed by Won and Aluru [7] where obtained from a B3LYP computation performed on an isolated BNNT (capped at both ends with hydrogen atoms) as shown in Fig. 1 using the CHelpG method. Thus it is not surprising that their results closely resemble the G03 (B3LYP) results reported here. Comparison with the partial charges obtained by Nirmala and Kolandaivel [17] is more difficult. They used partially capped structures and population analysis-based schemes. Their paper seems to suggest that the values computed using NPA are most reliable. These are at the higher end of the ranges given in Table 1. These results suggest, however, that such large charges will poorly describe the electrostatic potential in hollow BNNT, and this should be true both for an isolated and an infinite nanotube.

Adding water to the system leads to significantly larger charges on both the boron and nitrogen atoms. This is in line with the expectation that polarisation effects will be important in BNNT, although as demonstrated above it could also be due to changes in the number of grid points within the tube. In this case computation of the partial charges using only inner (2000 points) or outer (35000 points) grid points has much less effect than for the hollow BNNT system.

Interestingly, while for the system without water the charges on boron and nitrogen computed using CPMD are smaller than those computed using G03 (BLYP), the opposite is true for the system with water. This is probably because of basis set superposition effects that allow the boron and nitrogen atoms to benefit from the presence of basis functions on the $\mathrm{H}$ and $\mathrm{O}$ atoms when the water molecules are added to the system. Such effects do not occur in the CPMD calculations where the plane-wave basis set is the same for both computations. In any event this difference is small compared to the major change that occurs when adding water to the system. The results using B3LYP are again similar to BLYP. Overall these results are very similar to those reported by Won and Aluru [7].

To further examine the effect of water on the BNNT five of the water molecules were removed from the one with water system leaving just one water that is located roughly midway along the channel. The partial charges on the various rings of boron and nitrogen atoms that make up the nanotube are plotted in Fig. $2 a$. As expected, the charges at the centre of 

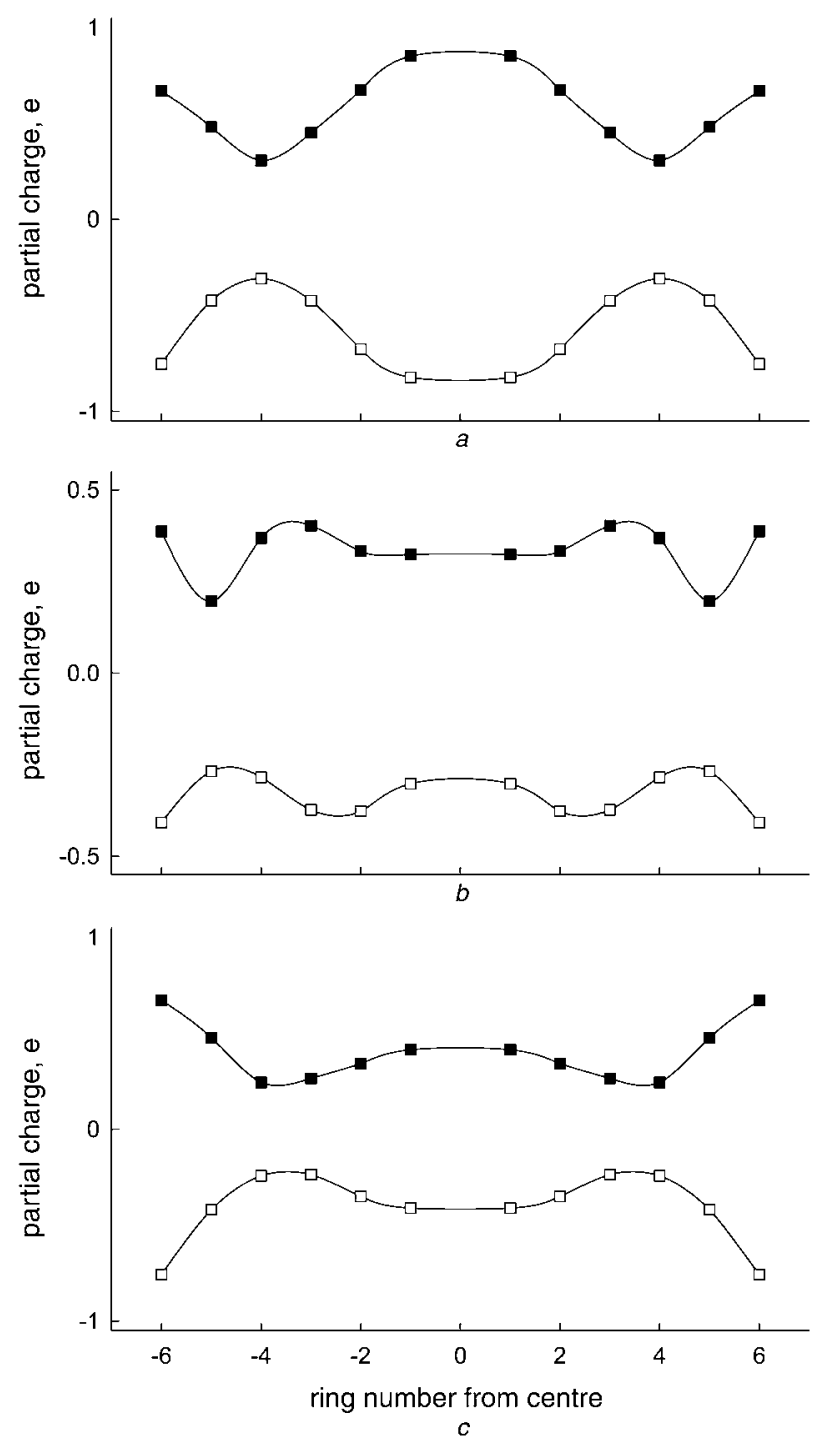

Figure 2 Partial charges on $B$ (solid squares) and $N$ (open squares) for the BNNT system as a function of ring number from the centre of the molecule computed using Gaussian03 with the CHelpG scheme

$a$ BNNT with one water and default grid for this system

$b$ BNNT with no water, but using the same grid as in Fig. 2(a)

$c$ BNNT with no water and the default grid for this system

the tube have increased substantially. To determine what fraction of this increase is owing to the use of different grid points, the authors also computed the charge values for this system using the same grid but without the water molecule (Fig. 2b) as well as using the default grid for the hollow BNNT (Fig. 2c). The results for the two hollow systems show differences, but these are small compared to the changes induced by placing a water molecule inside the tube. Overall, it appears that there is significant polarisation of the BNNT to a distance of about $3 \AA$ in either direction from the water molecule.

The overall objective of computing charges on the boron and nitrogen atoms was to obtain values that could be used as part of a classical force field that describes correctly the interaction between water and BNNT. In the process, it was shown that even though grid-based methods are generally considered to more accurately represent the partial charges on atom centres in a molecule, one needs to be careful with systems such as BNNT that have a hollow tube structure, as the position of grid points inside or outside the tube can greatly affect the computed partial charges.

To test the accuracy of the force field when using the above charges, the interaction energy of each of the water molecules with the nanotube for the 'with-water' structure were computed. Each water molecule was represented using TIP3P. As all the partial charges computed on boron and nitrogen for the 'with-water' BNNT system are similar, here only the results obtained when using the charges of Won and Aluru [7] are reported. These charge values, together with the values for $\varepsilon$ and $\sigma$ that were used by Won and Aluru [7] are collected together in Table 2. To explore the difference in the interaction energies the authors fixed the structure of the BNNT and created three configurations with random orientation of six water molecules within the tube. The resulting interaction energies for each of the 18 water molecules using these force field parameters are compared with those obtained using Grimme [25] corrected CPMD in Fig. 3.

The results in Fig. 3 show a significant difference between the CPMD results and those from the classical force field. This suggests either poor parameterisation of the force field or a fundamental inability of the force field to describe correctly this system. For each of the 18 water molecules, the interaction energy with the nanotube was computed using the Grimme corrected CPMD method. These interaction energies were then compared with the corresponding force field energies and the values of $q, \varepsilon$ and $\sigma$ for boron and nitrogen were allowed to vary in order to minimise the difference. The new set of optimised parameters are given in Table 2, with interaction energies for the same 'with water' nanotube system given in Fig. 3.

The re-parameterised results in Fig. 3 give much improved interaction energies compared to the Won and Aluru [7] force field parameters. Comparing the two sets of parameters given in Table 2, the values of the partial charges are very similar and appear reasonable given the variation reported in Table 1 . The most notable difference

Table 2 Partial charges $q(e)$ and the Lennard-Jones parameters $\varepsilon(\mathrm{kcal} / \mathrm{mol})$ and $\sigma(\AA)$ used by Won and Aluru [6, 7] for $B$ and $N$ atoms, compared to those derived by fitting to water/BNNT interaction energies

\begin{tabular}{|c|c|c|c|c|c|c|}
\hline & \multicolumn{3}{|c|}{ From [6, 7] } & \multicolumn{3}{c|}{ This work } \\
\cline { 2 - 7 } & $q$ & $\varepsilon$ & $\sigma$ & $q$ & $\varepsilon$ & $\sigma$ \\
\hline B & 1.05 & 0.0949 & 3.453 & 0.975 & 0.4530 & 3.380 \\
\hline N & -1.05 & 0.145 & 3.365 & -0.975 & 0.2030 & 3.215 \\
\hline
\end{tabular}




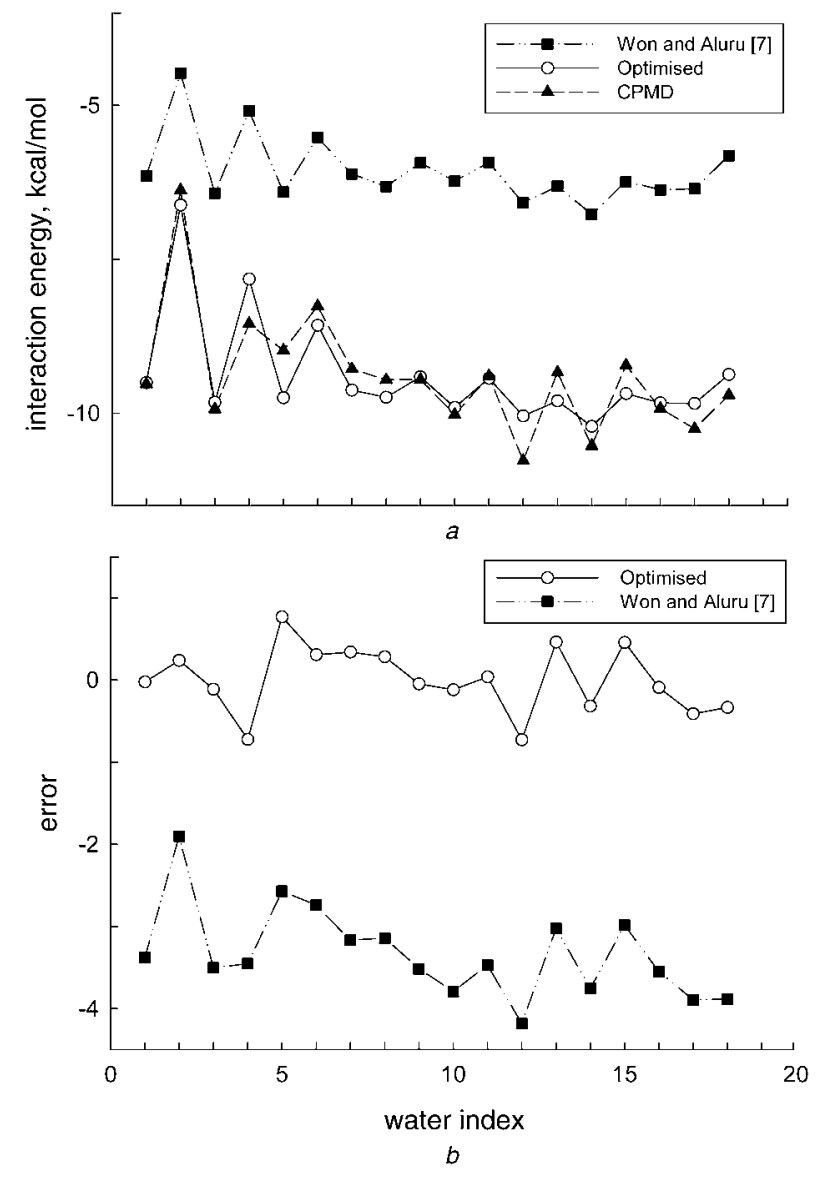

Figure 3 Comparison of results with Won and Aluru [7]

a Interaction energy calculated from CPMD, Won and Aluru [7] and re-parameterised force field

$b$ Relative error between CPMD interaction energies and those calculated with the other two force fields

The re-parameterised force field values are in Table 2 (This work), and the relative error in $b$ is calculated by subtracting the interaction energy determined in [7] or by re-parameterised force field from that determined from CPMD

is in the values for $\varepsilon$, and this is especially true for boron where the re-parameterised value is approximately five times larger than the value used by Won and Aluru [7]. Larger values for $\varepsilon$ will give deeper Lennard-Jones interaction potentials, whereas the slightly larger values for $\sigma$ will also cause the minimum in the potential to move to slightly shorter distances.

\section{Conclusions}

The partial charges computed here clearly indicate a significant shift in electron density between the boron and nitrogen atoms in BNNT. Moreover, this movement of charge increases dramatically when water is placed within the nanotube. Force fields that fail to assign charges to the boron and nitrogen atoms are clearly unphysical, whereas force fields that use fixed charges are likely to require the values of the partial charges to be parameterised according to the species the nanotube is interacting with. Even then they may be unable to describe well the processes that involve changes in interactions, such as the entry of a single water molecule into an empty nanotube.

This Letter has also shown that care needs to be taken when deriving partial charges using grid-based schemes for BNNT systems as the results can vary greatly depending on whether grid points are located inside or outside the nanotube. Thus, comparisons between partial charges computed for a hollow BNNT system with those computed for an 'occupied' BNNT system should be mindful of differences that can result purely from the removal of grid points that are within the nanotube.

When using a force field that combined charges obtained from quantum mechanics with existing Lennard-Jones parameters the authors were unable to reproduce the interaction energies of water molecules with the nanotube as computed using DFT theory corrected for long-range dispersion effects. This was true for all reasonable charge values. Taking an alternative approach where the charges and Lennard-Jones parameters for boron and nitrogen were adjusted to fit the interaction energies led to much better agreement. The largest difference between the fitted parameters and those previously used is for the value of $\varepsilon$ for boron, which is about five times larger. Calculations are now underway to investigate the effect of using these new parameters in MD simulations of BNNT, particularly in comparison to limited CPMD computations.

\section{Acknowledgments}

The authors gratefully acknowledge the support from the National Health and Medical Research Council and the Australia Research Council for grant LP0774896. This research was undertaken on the NCI National Facility in Canberra, Australia, which is supported by the Australian Commonwealth Government. The authors thank Silvie Ngo and Rhys Hawkins.

\section{References}

[1] HILDER T.A., GORDON D., CHUNG S.H.: 'Salt rejection and water transport through boron nitride nanotubes', Small, 2009, 5, pp. 2183-2190

[2] SUK M.E., RAghUnATHAN A.V., ALURU N.R.: 'Fast reverse osmosis using boron nitride and carbon nanotubes', Appl. Phys. Lett., 2008, 92, article no. 133120

[3] KANG J.W., HWANG H.J.: 'Comparison of C60 encapsulations into carbon and boron nitride nanotubes', J. Phys.: Condens. Matter, 2004, 16, pp. 3901-3908

[4] CHEN X., WU P., ROUSSEAS M., ET AL.: 'Boron nitride nanotubes are noncytotoxic and can be functionalized for interaction with proteins and cells', J. Am. Chem. Soc., 2009, 131, pp. 890-891 
[5] ISHIGAMI M., ALONI S., ZETTL A.: 'Properties of boron nitride nanotubes'. AIP Conf. Proc. for the 12th Int. Conf. STM'03, 2003, vol. 696, p. 94

[6] WON C.Y., ALURU N.R.: 'Water permeation through a subnanometer boron nitride nanotube', J. Am. Chem. Soc., 2007, 129, pp. 2748-2749

[7] WON C.Y., ALURU N.R.: 'Structure and dynamics of water confined in a boron nitride nanotube', J. Phys. Chem. C, 2008, 112, pp. $1812-1818$

[8] LU D., LI Y., RaVaiolo U., SCHUlten K.: 'Empirical nanotube model for biological applications', J. Phys. Chem. B, 2005, 109, pp. 11461-11467

[9] WON C.Y., JOSEPH S., ALURU N.R.: 'Effect of quantum partial charges on the structure and dynamics of water in singlewalled carbon nanotubes', J. Chem. Phys., 2006, 125, article no. 114701

[10] MANN D.J., HALLS M.D.: 'Water alignment and proton conduction inside carbon nanotubes', Phys. Rev. Lett., 2003, 90, article no. 195503

[11] BENEDICT L.X., LOUIE S.G., COHEN M.L.: 'Static polarizabilities of single-wall carbon nanotubes', Phys. Rev. B, 1995, 52, pp. $8541-8549$

[12] MOULIN F., DEVEL M., PICAUd S.: 'Molecular dynamics simulations of polarizable nanotubes interacting with water', Phys. Rev. B, 2005, 71, article no. 165401

[13] BRENEMAN C.M., WIBERG K.B.: 'Determining atom-centered monopoles from molecular electrostatic potentials. The need for high sampling density in formamide conformational analysis', J. Comput. Chem., 1990, 11, pp. 361-373

[14] BeSLeR B.H., MERZ JR. K.M., KOLLMAN P.A.: 'Atomic charges derived from semiempirical methods', J. Comput. Chem., 1990, 11, pp. 431-439

[15] SINGH U.C., KOLLMAN P.A.: 'An approach to computing electrostatic charges for molecules', J. Comput. Chem., 1984, 5, pp. $129-145$

[16] CHIRLIAN L.E., FRANCL M.M.: 'Atomic charges derived from electrostatic potentials: a detailed study', J. Comput. Chem., 1987, 8, pp. 894-905
[17] NIRMALA V., KOLANDAIVEL P.: 'Structure and electronic properties of armchair boron nitride nanotubes', J. Mol. Struct. THEOCHEM, 2007, 817, pp. 137-145

[18] REED A.E., WEINHOLD F.: 'Natural bond orbital analysis of near-Hartree-Fock water dimer', J. Chem. Phys., 1983, 78, pp. 4066-4073

[19] MAYO S.L., OLAFSON B.D., GODDARD III W.A.: 'DREIDING: a generic force field for molecular simulations', J. Phys. Chem., 1990, 94, pp. 8897-8909

[20] RAPPÉ A.K., CASEWIT C.J., COLWELL K.S., GODDARD III W.A., SKIFF W.M.: 'UFF, a full periodic table force field for molecular mechanics and molecular dynamics simulations', J. Am. Chem. Soc., 1992, 114, pp. 10024-10035

[21] KALE L., SKEEL R., BHANDARKAR M., ET AL.: 'NAMD2: greater scalability for parallel molecular dynamics', J. Comput. Phys., 1999, 151, pp. 283-312

[22] CPMD V3.13 Copyright IBM Corp 1990-2008, Copyright MPI fuer Festkoerperforschung, Stuttgart, 1997-2001

[23] FRISCH M.J., TRUCKS G.W., SCHLEGEL H.B., ET AL.: 'Gaussian 03, revision C.02' (Gaussian, Inc., Wallingford, CT, 2004)

[24] BOYS S.F., BERNARDI F.: 'The calculation of small molecular interactions by the differences of separate total energies. Some procedures with reduced errors', Mol. Phys., 1970, 19, pp. 553-566

[25] GRIMME S.: 'Semiempirical GGA-type density functional constructed with a long-range dispersion correction', J. Comput. Chem., 2006, 27, pp. 1787-1799

[26] WIDMAYER P., BOYEN H.-G., ZIEMANN P., REINKE P., OELHAFEN P.: 'Electron spectroscopy on boron nitride thin films: comparison of near-surface to bulk electronic properties', Phys. Rev. B: Condens. Matter Mater. Phys., 1999, 59, pp. 5233-5241

[27] HUMPHREY W., DALKE A., SCHULTEN K.: 'VMD: visual molecular dynamics', J. Mol. Graph., 1996, 14, pp. 33-38

[28] PARK N., CHO J., NAKAMURA H.: 'First-principles calculations on boron nitride nanotubes', J. Phys. Soc. Jpn., 2004, 73, pp. 2469-2472 